\title{
Review of Stereotactic Radiosurgery for Intramedullary Spinal Lesions
}

\author{
Hyung-Ki Park, Jae-Chil Chang \\ Department of Neurosurgery, Soonchunhyang University College of Medicine, Seoul, Korea
}

Stereotactic radiosurgery (SR) represents an increasingly utilized modality in the treatment of intracranial and extracranial pathologies. Stereotactic spine radiosurgery (SSR) uses an alternative strategy to increase the probability of local control by delivering large cumulative doses of radiation therapy (RT) in only a few fractions. SSR in the treatment of intramedullary lesions remains in its infancy - this review summarizes the current literature regarding the use of SSR for treating intramedullary spinal lesions. Several studies have suggested that SSR should be guided by the principles of intracranial radiosurgery with radiation doses placed no further than 1-2 mm apart, thereby minimizing exposure to the surrounding spinal cord and allowing for delivery of higher radiation doses to target areas. Maximum dose-volume relationships and single-point doses with SSR for the spinal cord are currently under debate. Prior reports of SR for intramedullary metastases, arteriovenous malformations, ependymomas, and hemangioblastomas demonstrated favorable outcomes. In the management of intrame- dullary spinal lesions, SSR appears to provide an effective and safe treatment compared to conventional RT. SSR should likely be utilized for select patient-scenarios given the potential for radiation-induced myelopathy, though high-quality literature on SSR for intramedullary lesions remains limited.

Key Words: Stereotactic radiosurgery $\cdot$ Spine $\cdot$ Intramedullary lesion

\section{INTRODUCTION}

Stereotactic radiosurgery (SR) represents an increasingly utilized treatment for intracranial and extracranial pathologies. Currently, SR is considered a therapeutic adjuvant though its role as a primary intervention continues to be explored.. SR was first conceived by Lars Leksell ${ }^{18)}$ as a potential tissue ablation modality in intracranial functional neurosurgery. Further improvements in frameless stereotactic technology allowed for advanced targeting capabilities of spinal lesions. ${ }^{1)}$ During the mid-1990s, investigators sought to expand the scope of SR to extracranial pathology-specifically on spinal tumors. In 1996, Hamilton et al. ${ }^{12)}$ first reported the use of SR for spinal pathology using a linear accelerator (LINAC). More recently, SR was defined as using externally generated ionizing radiation to inactivate or eradicate defined targets in the head or spine performed in a limited number of sessions (up to a maximum of five $)^{2)}$. A recent survey of 551 practitioners of SR highlighted the broad use of stereotactic body radiotherapy

- Received: December 1, 2012 • Revised: December 22, 2012

- Accepted: February 1, 2013

Corresponding Author: Hyung-Ki Park, MD

Department of Neurosurgery, Soonchunhyang University Hospital, 657

Hannam-dong, Yongsan-Gu, Seoul 140-743, Korea

Tel: +82-2-709-9268, Fax: +82-2-792-5976

E-mail:phk007@schmc.ac.kr
(SBRT) in clinical practice in the United States with $67.5 \%$ of survey-responders reportedly treating spinal pathology through SBRT ${ }^{22)}$.

Modern microsurgical techniques allow for safe resection of intramedullary spinal tumors ${ }^{8)}$. Postoperative radiation therapy (RT) may aid in reducing tumor recurrence and improving long-term survival in spinal cord ependymomas and astrocytomas $^{14,17}$. Conventional RT is commonly utilized in the treat ment of spinal cord tumors, especially in conjunction with surgical resection ${ }^{7}$. Stereotactic spine radiosurgery (SSR) uses an alternative strategy to conventional RT in an effort to help improve local control by delivering large cumulative doses of $\mathrm{RT}$ in fewer fractions ${ }^{25,37}$. Select patient populations may benefit most from SR including those with intramedullary spinal lesions. For example, Ryu et al. ${ }^{27}$ presented intriguing data on intramedullary spinal tumors treated with SSR-however, comprehensive data of SSR for spinal lesions requires further study.

This review article will summarize the use of SSR for intramedullary spinal lesions.

\section{Stereotactic Radiosurgery and Stereotactic Radiotherapy}

No consensus definition of radiosurgery exists among neurosurgeons and radiation oncologists. Multiple reports have 
attempted to clarify, define, or redefine the terms stereotactic radiosurgery (SR) and stereotactic radiotherapy $(S R T)^{2}$.

Modern LINAC technology is equipped for a wide variety of treatment modalities including intensity-modulated radiation therapy, stereotactic management, and image-guided radiation therapy. Advancements in LINAC technology have made hypofractionation more feasible and have reduced the toxicities associated with administering large fraction sizes ${ }^{11}$. SBRT can deliver high, ablative radiation doses (typically $>5$ Gy per fraction) in a limited number of fractions (1-5 fractions) to $\geq 1$ extracranial target(s) $)^{3,6,22,28,36}$.

Historically, SR treatment involved the delivery of a single radiation fraction. However, in 1996, the American Association of Neurological Surgeons (AANS) and the Congress of Neurological Surgeons (CNS) redefined radiosurgery, stating that: SR typically is performed in a single session, using a rigidly attached stereotactic guiding device, other immobilization technology and/or a stereotactic image-guidance system, but can be performed in a limited number of sessions, up to a maximum of five $e^{2)}$. However, confusion remains between the terminologies SBRT and SSR, in large part because the term SBRT originated from RT and SSR. Neurosurgeons tend to use SSR over SBRT-therefore, we will use SSR henceforth in this review.

\section{Current Systems for SSR}

Similar to intracranial SR, SSR delivers an ablative dose of conformal radiation to the target volume with a steep fall-off in dose beyond the treatment region. The development of Gamma-knife radiosurgery and LINAC-based techniques allowed for the delivery of highly conformal doses of radiation within a single fraction ${ }^{6}$. CyberKnife prototypes from Accuray were first used in the 1990s, and, in 2001, the FDA granted clearance for their use in treating extracranial lesions ${ }^{21)}$. Published reports have suggested that SSR specifications should be guided by the requirements of intracranial radiosurgery - with doses placed no further than 1-2 mm apart ${ }^{28)}$. The most commonly utilized SSR machines include Elekta Synergy S, Novalis (Brainlab) and CyberKnife (Table 1)-all employ computed-tomographybased technology for treatment planning. Novalis and Cyber-
Knife also use serial radiographs during SR treatment to account for spine movement and adjust treatment accordingly. Elekta Synergy $S$ and Novalis utilize a mobile table to change targeting coordinates, while CyberKnife uses a mobile robotic arm. Data suggest that all systems have excellent accuracy, and additional studies indicate that targeting areas remain accurate to within $1 \mathrm{~mm}^{5,15,38)}$. For example, CyberKnife was found to have a clini cally relevant accuracy of $0.7-0.3 \mathrm{~mm}^{5}$. Overall, such systems minimize radiation exposure to the spinal cord and allow for the relatively safe application of high doses of radiation to target $\operatorname{areas}^{39)}$.

\section{Radiobiology of SSR}

A main advantage of SSR over conventional RT lies in the higher biological effective dose (BED) delivered to the tumor or lesion. Through the highly conformal dose-shape surrounding the tumor target, the volume of non-tumor tissue not exposed to radiation is significantly lower in SSR. This aids in reducing acute-onset and late-onset radiation-induced complications and allows for more efficacious, higher target doses for radioresistant tumors $13,28,33)$.

The linear-quadratic model is widely accepted as a predictive tool for quantifying the effects of ionizing radiation on cells and often serves as the basis for determining fractionation schemes ${ }^{\text {). }}$. In general, this model suggests that the severity of the late-responding effects on tissues such as the spinal cord increases with larger fraction sizes. The $\alpha$ and $\beta$, respectively, represent coefficients for the non-repairable and repairable components of radiation-induced tissue damage. The constants $\alpha$ and $\beta$ are defined as the dose where $\alpha$ effects and $\beta$ effects equal one another. Tissues such as the spinal cord are thought to have a lower $\alpha / \beta$ ratio (at 3 Gy or less) - a setting where $\beta$ effects predominate or, said differently, an environment with less irreparable damage and/or greater capacity to repair radiation damage $)^{4)}$. This finding may be attributable, in part, to the low rate of mitosis that neural tissue undergoes or secondary to other repair mechanisms that allow for a greater capacity to repair radiation damage ${ }^{4)}$. Of note, radioresistant tumors like melanoma and sarcoma are believed to have

Table 1. Commercially available radiation therapy technologies

\begin{tabular}{ll}
\hline \hline System Name (Company Name) & \multicolumn{1}{c}{ System Characteristics } \\
\hline Novalis (Brainlab) & Optical tracking of infrared sensitive markers \\
TomoTherapy (Accuray) & CT image guidance \\
CyberKnife (Accuray) & Enhanced Xsight spine tracking \\
Elekta Synergy S (Elekta) & Integrated kilovoltage $(\mathrm{kV})$ X-ray capable of cone beam CT image production \\
\hline CT=computer-tomography &
\end{tabular}


smaller $\alpha / \beta$ values ${ }^{6}$.

Although the linear-quadratic model has limitations - including overestimated cell destruction ${ }^{10)}$ - it provides valuable information about tumor control and normal tissue toxicity ${ }^{19}$. Radiation triggers a multitude of cellular effects that result in cell death outside the mitotic pathway. Cellular apoptosis is an important component of such processes with research indicating that endothelial apoptosis becomes significant above a single-dose threshold of 8-10 Gy ${ }^{6}$. Radiation effects on tumor vasculature and tumor hypoxia also likely play a role in tumor responsiveness. Other critical mechanisms of cellular and tissue behavior that contribute to radiation response have yet to be elucidated.

SR appears particularly suitable for parallel, glandular organs like lung, kidney, and liver with structurally distinct subunits $^{6}$. Serial functioning tissues from linear or branching organs like the spinal cord, esophagus, bronchi, and bowel with undefined subunits may also benefit from reduced high-dose volume though the applications are less studied. Concern exists for ablating tissue like the spinal cord given the potential for irreversible downstream effects following damage to upstream portions of the organ ${ }^{6}$. Small volumes of serially functioning tissues such as the spinal cord likely can receive suprathreshold radiation doses, though the appropriate volume and anatomical regions to safely target have not been well characterized nor has the impact of inhomogeneous dose delivery ${ }^{6,16,19}$.

Permanent, radiation-induced myelopathy signifies the gravest concern for using SSR in intramedullary spinal lesions. When it occurs, permanent radiation-induced myelopathy typically has severe effects as normal tissues are more sensitive to the high-dose-per-fraction radiation. Case reports have documented paralysis secondary to permanent radiation-induced myelopathy - underscoring a devastating outcome associated with this technique ${ }^{6,13,20,24,29)}$.

\section{Dosimetric Considerations in the Spinal Cord}

Radiation-induced myelopathy has been reported in patients treated with SSR but with no history of prior radiation exposure $^{30)}$. This is a significant consideration when choosing SSR as the risk of radiation-induced myelopathy for conventional RT is nearly zero with $30 \mathrm{~Gy}$ of radiation delivered in 10 fractions. For fractionated radiation at $2 \mathrm{~Gy}$ per fraction, a homogenous dose of $45 \mathrm{~Gy}$ results in less than a $0.5 \%$ risk of radiation-induced myelopathy ${ }^{32)}$. For single fraction radiotherapy, spinal cord tissue tolerance remains unknown but has been estimated at 8-10 Gy for homogeneous exposure ${ }^{32}$. Interestingly, the cauda equina appears to be more tolerant than the spinal cord to SSR-similar to conventionally fractionated radiation-though the SSR doses that result in cauda equina injury have not been fully characterized ${ }^{6,32)}$.

Limits on spinal cord dosing have been published based mos tly on 5 cases of radiation-induced myelopathy with SSR. In those patients, the thecal sac was defined as the avoidance structure. Radiation-induced myelopathy was observed at maxi mum point doses of $25.6 \mathrm{~Gy}$ in 2 fractions, $30.9 \mathrm{~Gy}$ in 3 fractions as well as $14.8,13.1$, and $10.6 \mathrm{~Gy}$ in 1 fraction $^{30,31)}$. The authors concluded that $10 \mathrm{~Gy}$ in a single fraction was safe and suggested that a normalized 2-Gy-equivalent BED of $35 \mathrm{~Gy}$ delivered in up to 5 fractions carried a low risk of radiation-induced myelopathy ${ }^{30)}$. This translated to a maximum dose of $14.5 \mathrm{~Gy}$ in 2 fractions, $17.5 \mathrm{~Gy}$ in 3 fractions, 20 Gy in 4 fractions, and $22 \mathrm{~Gy}$ in 5 fractions administered to a point within the thecal $\mathrm{sac}^{16,30)}$. According to recent data on SSR, a maximum spinal cord dose of $13 \mathrm{~Gy}$ in a single fraction or $20 \mathrm{~Gy}$ in 3 fractions appears to be associated with a $<1 \%$ risk of injury. ${ }^{16}$ The decision to use higher doses must weigh the benefit of tumor control against the potential for radiation toxicity. ${ }^{28)}$

The maximum dose-volume relationship and single-point dose tolerated by the spinal cord are unknown and currently under debate. Ryu et al. ${ }^{26}$ reported that the partial volume tolerance of the spinal cord is $\geq 10$ Gy to $10 \%$ of the total spinal cord volume (with spinal cord volume defined as $6 \mathrm{~mm}$ superior and inferior to the radiosurgery target). Dose constraints for SSR are described in the Radiation Therapy Oncology Group (RTOG) Protocol \#0631 comparing SR to conventional RT and are as follows: (1) for spinal cord (contoured, from a fused MRI scan, 5 to $6 \mathrm{~mm}$ cranial and caudal to the target), $10 \%$ to receive $<10 \mathrm{~Gy}, 0.35 \mathrm{~mL}<10 \mathrm{~Gy}$, and $0.035 \mathrm{~mL}<14 \mathrm{~Gy}$; and (2) for cauda equina, $5 \mathrm{~mL}<14 \mathrm{~Gy}$ and $0.035 \mathrm{~mL}<16 \mathrm{~Gy}$. However, insufficient long- term data prevent calculation of a dose-volume relationship for radiation-induced myelopathy when the partial cord was treated with a hypofractionated regimen ${ }^{6}$.

\section{Imaging, Treatment Planning and SSR Delivery}

Several features of SSR underscore the importance of appropriate imaging. First, SSR creates highly conformal dose distributions intended to target the tumor and avoid critical structures, thereby necessitating accurate target and normal tissue delineation. Second, steep dose gradients demonstrate that appropriate dosing can vary from an acceptable range to exceeding tolerance based on only 1-2 mm changes in distance ${ }^{28)}$. Taken together, SSR requires accurate pre-treatment planning and delivery, requirements serviced by high-quality imaging (Fig. 1).

A planning computed tomography (CT) scan is performed 


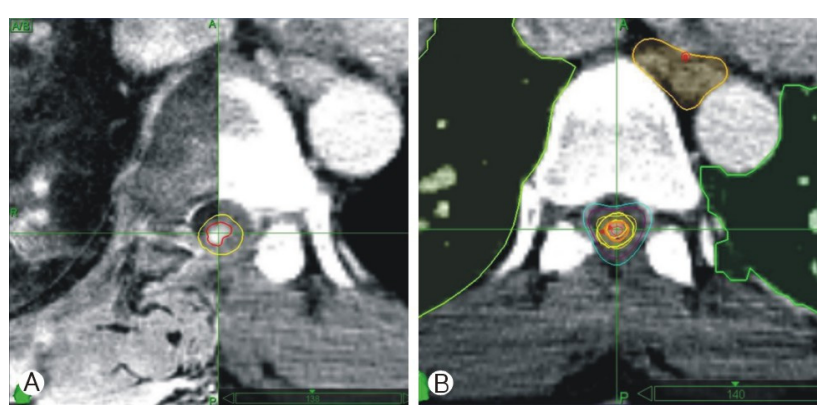

Fig. 1. Images from recurrent intramedullary metastatic cancer at T7 status-post resection, illustrating pre-treatment CT image fusion with MRI scan for contouring (A: CTV and spinal cord demarcated as red and yellow lines, respectively) as well as the ultimate planning image for treatment (B). Of note, radiation dose was 18 Gy in 3 fractions to the $80 \%$ isodose line.

with the patient in treatment position and immobilized, if necessary. CT slice thickness typically is $<3 \mathrm{~mm}$. Intravenous contrast can be used for improve tumor characterization however, CT is suboptimal for visualizing the spinal cord and intramedullary lesions as mean spinal cord volumes have been shown to be inaccurately larger with CT scans than with MRI data ${ }^{28)}$. CT-MRI image fusion further improves treatment planning, though the optimal approach to radiographically contour neural tissues in the spinal remains a matter of debate.

Gross tumor volume (GTV) is defined as the radiographically visible tumor based on the fused CT-MRI images ${ }^{6}$. In intramedullary lesions, no clinical target volume (CTV) is applied and, thus, CTV=GTV. Planning target volume (PTV) can be expanded by 2-3 $\mathrm{mm}$ beyond the CTV. CTV-PTV expansion accounts for potential errors in patient setup, organ motion, and any mechanical inaccuracy of image-guided treatment delivery. Therefore, CTV-PTV respects dose limits to the spinal cord while keeping within the goal to alleviate patient symptoms and minimize risk of radiation-induced myelopathy $y^{6}$.

On treatment day, patients should be placed supine on a vacuum bag. Setup images are then taken from the imageguided radiotherapy system with any errors corrected prior to treatment. Coregistered images are then evaluated by the treating physician. Repositioning is performed if there is $>2$ $\mathrm{mm}$ difference between pre-treatment images and treatment images and/or if there is rotation $>2$ degrees (depending on the geometric margins used in the treatment plan) ${ }^{6}$.

\section{Radiation-Induced Myelopathy after Spinal Radiosurgery}

Delayed myelopathy after radiosurgery is uncommon. Many articles cite a case series of 6 patients treated with radiosurgery who developed delayed myelopathy ${ }^{6,916}$. Myelopathic symptoms in all patients were initially managed with corticosteroids. Some patients also received a combination of vitamin E and pentoxifylline (Trental; Sanofi Aventis, Bridgewater, NJ), hyperbaric oxygen, gabapentin (Neurontin; Pfizer, New York), and/or physical therapy. Following treatment, 3 of the 6 patients' myelopathic symptoms improved, 2 patients reached a clinical plateau, and 1 patient progressed to paraplegia. Of the 3 patients who improved clinically, follow-up MRI scans revealed complete radiographic resolution of their spinal cord edema".

\section{Clinical Outcomes}

\section{Spinal Metastasis}

In 2009, Parikh and Heron ${ }^{23)}$ reported a case of metastatic renal cell carcinoma in the intramedullary area of $\mathrm{C} 5$ treated with SSR using a dose of $15 \mathrm{~Gy}$ in 3 fractions to the $80 \%$ isodose line. At 26 months following treatment, the patient was alive, fully functional, and reported no pain with rare paresthesias. In 2009, Shin et $\mathrm{al}^{34)}$. reported data from 4 intradural extramedullary spinal tumors and 7 intramedullary metastases all treated with SSR using image-guided and intensitymodulated radiation. Mean treatment dose was 13.8 Gy (range: 10-16 Gy), and median follow-up duration was 10 months. Of those patients with intramedullary metastases, 5 patients improved clinically, 1 patient was unchanged, and 1 patient was lost to follow-up. Table 2 includes further details on the studies mentioned above.

\section{Spinal AVM}

In 2006, Sinclair et al. ${ }^{35)}$ published 15 cases of intramedullary arteriovenous malformations (AVM) treated with CyberKnife technology - of those patients studied, 7 received embolization before radiosurgery. Mean dose of 20.5 Gy was delivered to the margin of the AVM nidus in 2-5 fractions to decrease the risk of radiation-related spinal cord damage. Up to 3 years post-radiosurgery follow-up data was available. Complete angiographic obliteration after radiosurgery was seen in 1 patient, and 4 patients showed evidence of residual AVM on angiography (although AVM volumes were significantly reduced). Remaining patients did not undergo final angiography but showed significant AVM volume reduction on MRI. None of the patients demonstrated evidence of hemorrhage or neurological deterioration attributable to SSR (Table 2). 
Table 2. Reports of stereotactic spinal radiosurgery (SSR) for intramedullary lesions

\begin{tabular}{|c|c|c|c|c|c|}
\hline Study (Year) & $\begin{array}{l}\text { \# of } \\
\text { patients } \\
\text { /lesions }\end{array}$ & Disease $(\mathrm{s})$ & $\begin{array}{c}\text { System \& } \\
\text { radiation } \\
\text { dose }(G y / F x)\end{array}$ & $\begin{array}{l}\text { Follow-up } \\
\text { (Months) }\end{array}$ & Outcomes \\
\hline Ryu et al. ${ }^{27)}(2003)$ & $7 / 10$ & $\begin{array}{l}\text { Hemangioblastoma }(n=7) \text {, } \\
\text { ependymoma }(n=3)\end{array}$ & $\begin{array}{l}\text { CyberKnife } \\
18-25 / 1-3\end{array}$ & $1-24$ & Improved $(n=2)$, stable $(n=4)$, declined $(n=1)$ \\
\hline Sinclair et al. ${ }^{35)}(2006)$ & $15 / 15$ & $\begin{array}{l}\text { Arteriovenous malformation } \\
\text { (AVM) }\end{array}$ & $\begin{array}{l}\text { CyberKnife } \\
20.5 / 2-5\end{array}$ & $3-59$ & $\begin{array}{l}\text { Size reduction }(n=13) \text {, completeobliteration } \\
(n=1) \text {, clinicallyimprovedorstabilized }(92.3 \%) \text {, } \\
\text { e-bleeding }(n=0)\end{array}$ \\
\hline Parikh et al. ${ }^{23)}$ (2009) & $1 / 1$ & Metastatic renal cell cancer & $\begin{array}{c}\text { CyberKnife } \\
15 / 3\end{array}$ & 26 & Clinical improvement $(n=1)$ \\
\hline Shin et al. $^{34)}(2009)$ & $6 / 6$ & $\begin{array}{l}\text { Metastasis (melanoma: } \mathrm{n}=1 \text {; } \\
\text { breastcancer: } \mathrm{n}=2 ; C A ; \\
\text { lungcancer: } \mathrm{n}=3 \text { glioma: } \mathrm{n}=1 \text { ) }\end{array}$ & $\begin{array}{l}\text { Novalis } \\
10-16 / 1\end{array}$ & $2.2-19.4$ & Clinical improvement $(n=5)$, unchanged $(n=1)$ \\
\hline
\end{tabular}

$\mathrm{Gy}=$ Gray unit; $F x=$ numbers of radiation fractions

\section{Hemangioblastoma and Ependymoma}

In 2003, Ryu et al. ${ }^{27)}$ discussed 7 hemangioblastomas and 3 ependymomas treated with CyberKnife. Patients had either recurrent tumors, undergone several previous surgeries, possessed medical contraindications to surgery, and/or declined open resection. Conformal treatment planning prescribed doses of 18-25 Gy to the lesions in 1-3 stages. No significant treatment-related complications were recorded. Mean radiographic and clinical follow-up duration was 12 months (range: 1-24 months). On follow-up neuroimaging, 1 ependymoma and 2 hemangioblastomas were smaller-remaining tumors remained stable in size (Table 2).

\section{CONCLUSION}

In the management of intramedullary spinal lesions, SSR appears to provide an effective and safe alternative treatment option to conventional RT. However, the available literature on SSR for intramedullary spinal lesions remains limited, and proper spinal cord dosing in SSR has yet to be clarified. Therefore, SSR should be employed for select cases given the increased potential for radiation-induced myelopathy with higher-dose-per-fraction radiation. SSR in the treatment of intramedullary spinal lesions will continue to improve over time as image-guided systems deliver safer and more effective radiation therapy.

\section{REFERENCES}

1. Adler JR Jr, Murphy MJ, Chang SD, Hancock SL: Image-guided robotic radiosurgery. Neurosurgery 44:1299-1306; discussion
1306-1307, 1999

2. Barnett GH, Linskey ME, Adler JR, Cozzens JW, Friedman WA, Heilbrun MP, et al: Stereotactic radiosurgery--an organized neurosurgery-sanctioned definition. J Neurosurg 106:1-5, 2007

3. Benedict SH, Yenice KM, Followill D, Galvin JM, Hinson W, Kavanagh B, et al: Stereotactic body radiation therapy: the report of AAPM task group 101. Med Phys 37:4078-4101, 2010

4. Bentzen SM, Thames HD, Travis EL, Ang KK, Van der Schueren E, Dewit L, et al: Direct estimation of latent time for radiation injury in late-responding normal tissues: gut, lung, and spinal cord. Int J Radiat Biol 55:27-43, 1989

5. Chang SD, Main W, Martin DP, Gibbs IC, Heilbrun MP: An analysis of the accuracy of the cyberKnife: a robotic frameless stereotactic radiosurgical system. Neurosurgery 52:140-146; discussion 146-147, 2003

6. Chawla S, Schell MC, Milano MT: Stereotactic body radiation for the spine: a review. Am J Clin Oncol, 2011

7. Dahele M, Zindler JD, Sanchez E, Verbakel WF, Kuijer JP, Slotman BJ, et al: Imaging for stereotactic spine radiotherapy: clinical considerations. Int J Radiat Oncol Biol Phys 81:321-330, 2011

8. Gezen F, Kahraman S, Canakci Z, Beduk A: Review of 36 cases of spinal cord meningioma. Spine (Phila Pa 1976) 25:727-731, 2000

9. Guerrero M, Li XA: Extending the linear-quadratic model for large fraction doses pertinent to stereotactic radiotherapy. Phys Med Biol 49:4825-4835, 2004

10. Haley ML, Gerszten PC, Heron DE, Chang YF, Atteberry DS, Burton SA: Efficacy and cost-effectiveness analysis of external beam and stereotactic body radiation therapy in the treatment of spine metastases: a matched-pair analysis. J Neurosurg Spine 14:537-542, 2011

11. Hamilton AJ, Lulu BA, Fosmire H, Gossett L: LINAC-based spinal stereotactic radiosurgery. Stereotact Funct Neurosurg 66: 1-9, 1996

12. Hsu W, Nguyen T, Kleinberg L, Ford EC, Rigamonti D, Gokaslan $\mathrm{ZL}$, et al: Stereotactic radiosurgery for spine tumors: review of current literature. Stereotact Funct Neurosurg 88:315-321, 2010

13. Isaacson SR: Radiation therapy and the management of intra- 
medullary spinal cord tumors. J Neurooncol 47:231-238, 2000

14. Kim S, Jin $\mathrm{H}$, Yang $\mathrm{H}$, Amdur RJ: A study on target positioning error and its impact on dose variation in image-guided stereotactic body radiotherapy for the spine. Int J Radiat Oncol Biol Phys 73:1574-1579, 2009

15. Kirkpatrick JP, van der Kogel AJ, Schultheiss TE: Radiation dose-volume effects in the spinal cord. Int J Radiat Oncol Biol Phys 76:S42-49, 2010

16. Kopelson G, Linggood RM, Kleinman GM, Doucette J, Wang CC: Management of intramedullary spinal cord tumors. Radiology 135:473-479, 1980

17. LEKSELL L: The stereotaxic method and radiosurgery of the brain. Acta Chir Scand 102:316-319, 1951

18. Milano MT, Constine LS, Okunieff P: Normal tissue toxicity after small field hypofractionated stereotactic body radiation. Radiat Oncol 3:36, 2008

19. Milano MT, Usuki KY, Walter KA, Clark D, Schell MC: Stereotactic radiosurgery and hypofractionated stereotactic radiotherapy: normal tissue dose constraints of the central nervous system. Cancer Treat Rev 37:567-578, 2011

20. Nomura R, Suzuki I: [CyberKnife radiosurgery--present status and future prospect]. Brain Nerve 63:195-202, 2011

21. Pan H, Simpson DR, Mell LK, Mundt AJ, Lawson JD: A survey of stereotactic body radiotherapy use in the united states. Cancer 117:4566-4572, 2011

22. Parikh S, Heron DE: Fractionated radiosurgical management of intramedullary spinal cord metastasis: a case report and review of the literature. Clin Neurol Neurosurg 111:858-861, 2009

23. Pirzkall A, Lohr F, Rhein B, Hoss A, Schlegel W, Wannenmacher $\mathrm{M}$, et al: Conformal radiotherapy of challenging paraspinal tumors using a multiple arc segment technique. Int J Radiat Oncol Biol Phys 48:1197-1204, 2000

24. Rades D, Lange M, Veninga T, Stalpers LJ, Bajrovic A, Adamietz IA, et al: Final results of a prospective study comparing the local control of short-course and long-course radiotherapy for metastatic spinal cord compression. Int J Radiat Oncol Biol Phys 79:524-530, 2011

25. Ryu S, Jin JY, Jin R, Rock J, Ajlouni M, Movsas B, et al: Partial volume tolerance of the spinal cord and complications of singledose radiosurgery. Cancer 109:628-636, 2007

26. Ryu SI, Kim DH, Chang SD: Stereotactic radiosurgery for hemangiomas and ependymomas of the spinal cord. Neurosurg
Focus 15:E10, 2003

27. Sahgal A, Bilsky M, Chang EL, Ma L, Yamada Y, Rhines LD, et al: Stereotactic body radiotherapy for spinal metastases: current status, with a focus on its application in the postoperative patient. J Neurosurg Spine 14:151-166, 2011

28. Sahgal A, Larson DA, Chang EL: Stereotactic body radiosurgery for spinal metastases: a critical review. Int J Radiat Oncol Biol Phys 71:652-665, 2008

29. Sahgal A, Ma L, Gibbs I, Gerszten PC, Ryu S, Soltys S, et al: Spinal cord tolerance for stereotactic body radiotherapy. Int J Radiat Oncol Biol Phys 77:548-553, 2010

30. Sahgal A, Ma L, Weinberg V, Gibbs IC, Chao S, Chang UK, et al: Reirradiation human spinal cord tolerance for stereotactic body radiotherapy. Int J Radiat Oncol Biol Phys 82:107-116, 2012

31. Schultheiss TE, Kun LE, Ang KK, Stephens LC: Radiation response of the central nervous system. Int J Radiat Oncol Biol Phys 31:1093-1112, 1995

32. Sheehan JP, Shaffrey CI, Schlesinger D, Williams BJ, Arlet V, Larner J: Radiosurgery in the treatment of spinal metastases: tumor control, survival, and quality of life after helical tomotherapy. Neurosurgery 65:1052-1061; discussion 1061-1062, 2009

33. Shin DA, Huh R, Chung SS, Rock J, Ryu S: Stereotactic spine radiosurgery for intradural and intramedullary metastasis. Neurosurg Focus 27:E10, 2009

34. Sinclair J, Chang SD, Gibbs IC, Adler JR Jr: Multisession CyberKnife radiosurgery for intramedullary spinal cord arteriovenous malformations. Neurosurgery 58:1081-1089; discussion 10811089, 2006

35. Sohn S, Chung CK: The role of stereotactic radiosurgery in metastasis to the spine. J Korean Neurosurg Soc 51:1-7, 2012

36. Timmerman RD, Kavanagh BD, Cho LC, Papiez L, Xing L: Stereotactic body radiation therapy in multiple organ sites. J Clin Oncol 25:947-952, 2007

37. Yan $\mathrm{H}$, Yin FF, Kim JH: A phantom study on the positioning accuracy of the novalis body system. Med Phys 30:3052-3060, 2003

38. Yu C, Main W, Taylor D, Kuduvalli G, Apuzzo ML, Adler JR Jr: An anthropomorphic phantom study of the accuracy of cyberknife spinal radiosurgery. Neurosurgery 55:1138-1149, 2004 